\title{
The experience of epilepsy in later life: a qualitative exploration of illness representations
}

\author{
Haris Yennadiou ${ }^{\text {a, }}$, Emma Wolverson $^{\mathrm{a}}$ \\ ${ }^{a}$ The University of Hull, Faculty of Health and Social Care. Aire Building, Hull, Cottingham \\ Road, Hull, HU6 7RX, UK \\ E-Mails: hyennadiou@nhs.net, e.wolverson@hull.ac.uk
}

\footnotetext{
${ }^{1}$ Corresponding author

Present address: Central and North West London NHS Trust, Clinical Health Psychology Department, Clarence Wing, St Mary's Hospital, London, W2 1NY, UK

E-mail: hyennadiou@nhs.net
} 


\section{Abstract}

The objective of this study is to explore how older people living with epilepsy appraise their condition through their lived-experience. The common-sense model of illness representations (CSMIR) provides a framework to explain how individuals make sense of and manage health threats. Semi-structured in-depth interviews based on the CSMIR were conducted with ten people with epilepsy who were above the age of 65 . The results were analysed using Interpretative Phenomenological Analysis. Three overarching themes emerged from the analysis: 'the power of epilepsy', 'they say you can live a normal life but you can't' and 'attempts to adjust and cope'. Epilepsy was described as a threatening, persistent and unpredictable condition associated with distressing experiences. Participants described a process of balancing negative psychosocial consequences including stigma, loss of control and reliance on other people and medication with parallel co-existing coping strategies. These attempts to manage the condition were characterised by a desire for acceptance and increased awareness of epilepsy, strategies to restore loss of control and strength derived from supportive relationships. We conclude that there is large scope for psychosocial interventions in healthcare provision for this patient group. The role of specialist nursing, relationshipcentred models, psychotherapy, educational and self-management programmes is highlighted.

\section{Keywords: older people; epilepsy; lived experience; illness representations; interpretative phenomenological analysis (IPA)}

\section{Introduction}

The prevalence of epilepsy in people above the age of 60 is higher than the general population, with incidence rising with increasing age [1]. Epilepsy in older age is associated with increased mortality and difficulties with diagnosis due to atypical symptoms that can mimic other conditions such as dementia $[2,3]$. Treatment can be complex for older people due to greater risk of antiepileptic medication side effects, physical health changes associated with normal ageing and an increased likelihood of medication interactions from the high prevalence of polypharmacy and co-morbidity in later life [4].

Despite these implications and in contrast to extensive literature looking at aspects of quality of life of working age adults with epilepsy [5], the psychosocial functioning of older people with the condition has received little attention. The available literature suggests that the psychological well-being of older people with epilepsy is poorer than the general population $[6,7,8]$. Notably, older people with epilepsy display significantly higher levels of depressive symptoms, higher levels of anxiety, more impaired sleep and lower cognitive status than agematched controls $[9,10]$.

Little is known about specific issues associated with the experience of epilepsy in later life and the impact on the individual's wellbeing. Seizures in older age may have different implications and consequences than in earlier stages of life [11]. In addition to coping with epilepsy older people are likely to face several other challenges and life transitions including retirement, bereavements, loss of role functioning and co-morbid physical health conditions. Studies to date revealed that older people living with epilepsy are affected by stigma which leads to nondisclosure of their diagnosis and difficulties in accepting their condition [12].

The evidence as to whether older people have a more impaired quality of life than younger adults with epilepsy is equivocal [6,8]. Interestingly, Pugh and colleagues report a more favourable health status profile for older people compared to middle-aged adults with epilepsy [13], suggesting that older people may be more resilient and have fewer social 
demands placed upon them. There is some evidence to suggest that there is similarity between the concerns of older people and working age adults with epilepsy, with driving and medication side effects being reported as the most significant concerns [14]. However, older people report more antiepileptic medication side effects which impact on quality of life [6].

The above findings are confounded by limited research in the field, methodological limitations including small samples $[8,9]$ and the absence of control groups in some studies [10]. Notably, the measures employed to assess quality of life are developed and validated for the general epilepsy population and therefore may not be sensitive to the issues and concerns of older people [11]. To our knowledge only two qualitative studies have explored psychosocial aspects of epilepsy in older people $[12,14]$, with one of the studies conducted employing structured analysis which arguably restricted the exploratory nature of the enquiry [14].

Qualitative research in epilepsy is increasingly recognised as integral for a more in-depth understanding of peoples' experiences of epilepsy [15]. One framework that can provide further insight into how older people appraise these experiences is the common-sense model of illness representations (CSMIR) $[16,17]$. The CSMIR proposes that people form illness representations from a range of sources including existing health and illness beliefs as well as their own experiences, to make sense of health challenges. Illness representations are constructed in five domains: illness identity or beliefs about the symptoms, time line, consequences, causes, and controllability or cure [17]. These illness constructs appear to influence a wide range of health and psychological outcomes including coping, well-being, and treatment adherence $[18,19]$. Work investigating the illness representations held by working age adults with epilepsy suggests that more negative illness representations and strong illness identity is associated with negative coping, poor adjustment and higher levels of anxiety [20,21].

The present qualitative study employed Interpretative Phenomenological Analysis (IPA) and aims to explore the lived experience of epilepsy in later life through older peoples' appraisals of their condition. IPA methodology provides a rich qualitative analysis and is widely used to understand and interpret how people make sense of illness experience through the use of language $[22,23]$.

\section{Methods}

\subsection{Participants}

Ten patients were recruited from a Neurosciences Department in the North of England with $34 \%$ respondent rate. This was deemed as an appropriate sample size based on the idiographic nature of IPA and guidance on its methodology [22]. Demographic characteristics and pseudonyms of the participants can be found in Table 1. Participants were White British/ English and above 65 years of age as this was the cut-off age for older adult health services in the UK at the time this study was conducted.

All participants had a confirmed diagnosis of epilepsy with a mean average of 23 years since diagnosis (range 2-48 years). For this exploratory study, no exclusion criteria were set in regard to age of epilepsy onset as the study was looking into the lived experience of epilepsy in later life rather than the differences between age of onset per se. Patients were excluded if they had a diagnosis of dementia, a learning disability, a serious co-existing terminal physical condition or a significant mental health condition that could influence their ability to share their experiences of epilepsy. Most participants were unsure about the type of epilepsy they 
were diagnosed with $(\mathrm{N}=6)$, two identified it as temporal-lobe epilepsy, one as postencephalitis epilepsy and one as 'petit mal'. All participants were taking at least one antiepileptic medication (range 1-3) and nine reported at least one co-existing health condition (range 1-5). Eight lived with their partner and two lived alone.

Table 1

Participants' demographic information

$\begin{array}{ll}\text { Participant Pseudonym } & \text { Age (Mean: } \\ & \text { 71.8, SD 4.24) }\end{array}$

\begin{tabular}{lll}
\hline Beth & 73 & Female \\
Ella & 69 & Female \\
Alfred & 81 & Male \\
Jennifer & 72 & Female \\
Ian & 70 & Male \\
Christine & 73 & Female \\
Carol & 68 & Female \\
Sarah & 67 & Female \\
Susan & 69 & Female \\
Alan & 76 & Male \\
\hline
\end{tabular}

3.2 Procedure

Patients who met inclusion criteria were identified by two specialist epilepsy nurses who provided an information sheet about the study. Those who contacted the researcher expressing a desire to participate were given a convenient meeting, time and location. A brief demographic questionnaire was completed; followed by the semi-structured in-depth interview lasting on average 57 minutes (range 38-79).

The interview schedule included open-ended questions guided by the components of the CSMIR [16,17]. This model was employed as it attempts to explain how individuals form beliefs and appraisals of a health challenge based on their experiences of that condition. The interview schedule also included a general question pursuing any other issues that participants felt were important (Appendix A). The interview questions were piloted and adapted following consultation with two epilepsy specialist nurses and two older people with epilepsy. The questions served as a guiding framework and did not restrict the exploration of themes to the constructs of the model. Instead, the interview was led by participants' narratives focusing on their priorities when describing their experiences.

\subsection{Analysis}

The interviews were digitally recorded, transcribed verbatim and analysed using the stages of IPA described by Smith and colleagues [22]. IPA was chosen as it endeavours to investigate how a person makes sense of a phenomenon under investigation through a close exploration of their 'lived experience' [22]. The process of this analysis requires the application on 'double hermeneutics' where the researcher attempts to interpret how the participant makes sense of their experience $[22,24]$. The main analysis was conducted by a female trainee clinical psychologist in her mid-twenties who had no personal experience of living with a long-term health condition. 
In the initial stages the researcher was 'immersed' in the data by carefully reading and rereading each transcript. During this process the researcher noted thoughts, comments and reflections that appeared significant in the margins of each transcript. The initial notes were then examined for connections that lead to emergent subordinate themes based on psychological concepts and resonant issues. The subordinate themes were structured in a table with illustrative quotes. Subsequently, meaningful connections and patterns between emergent themes were identified. Related themes were clustered together and were provided with an interpretative label leading to the development of super-ordinate, overarching themes. The process was repeated for each transcript with an emphasis on the idiographic nature of the analysis. A master table was then constructed capturing recurrent and salient subordinate and superordinate themes across participants. The process involved a constant review of the different analytic stages to ensure that the themes were grounded in the accounts.

This study employed the elements of an existing structural model to guide the development of the interview questions. However, the model was not used to guide the analysis. Themes were identified through an analytic process that maintained an exploratory nature and focused on how participants appraised their condition through their own lived experience, as defined by IPA methodology. Other studies employing structural models in IPA research have adopted a similar approach $[25,26]$.

\subsection{Quality and Validity}

Whilst there is no agreed method to ensure validity and quality of qualitative research [22], the second author (E.W.) followed an identical analysis process from coding samples of individual transcript analysis through to the final analysis and thematic categorisation. Where any questions arose about categorisation of themes, interpretative discussion took place until an agreement was reached. An IPA research group also examined and re-examined accounts and themes to validate thematic headings. A central focus of validation was ensuring that the themes were grounded in participants' verbatim. Themes were developed if they appeared prominent and resonated in accounts. No theme was excluded if it was not represented across the majority, to ensure that individual experiences were captured [22].

\subsection{Ethics}

Ethical approval was obtained via a local ethics committee at NHS Health Research Authority (12/EM/0227). The researcher obtained written informed consent from each participant at the start of each interview and confirmed confidentiality of their results. Participant anonymity was protected by assigning pseudonyms during data analysis and write-up.

\section{Results}

Three superordinate themes and eight subordinate themes emerged from the analysis. Figure 1 illustrates the themes and relationships between them. The superordinate theme, the power of epilepsy, dominated the whole experience and appeared to be the driving force between the other two co-existing and contrasting superordinate themes 'they say you can live a normal life but you can't' and attempts to adjust and cope. The powerful nature of the condition appeared to drive the negative consequences and pose a barrier to attempts for adjustment, leaving participants in a constant battle to balance the psychosocial challenges of epilepsy with various coping strategies. 


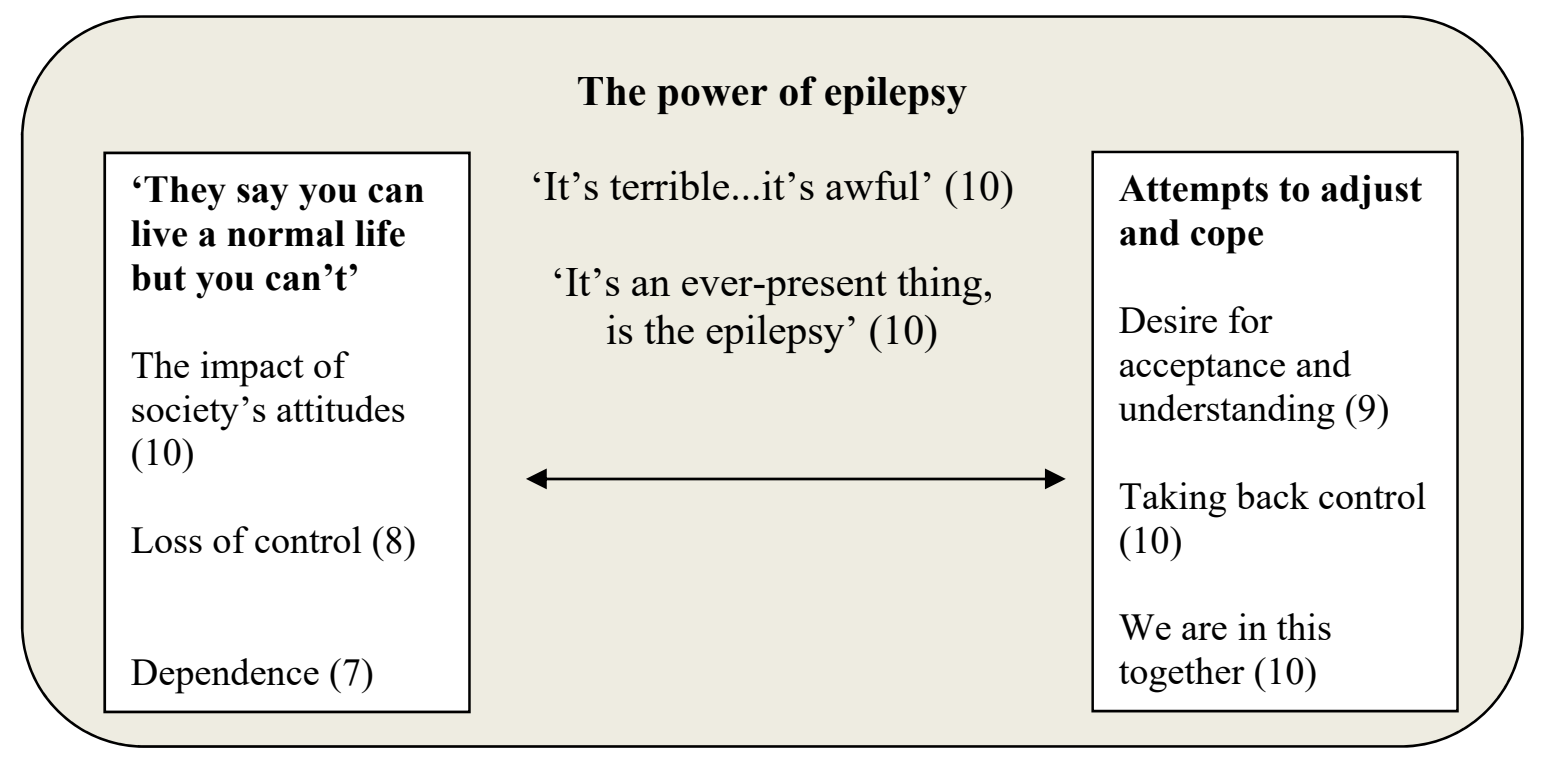

Fig. 1: An illustration of the relationships between the superordinate themes (in bold) and subordinate themes, with the number of participants supporting each subordinate theme in brackets.

\subsection{The Power of Epilepsy}

Throughout the accounts epilepsy was experienced as a powerful condition which was often referred to as 'it' and was described as a separate, threatening and unpredictable entity. Two subordinate themes reflected the traumatic, chronic and erratic nature of the condition.

\subsection{1 'It's terrible.... it's awful'}

Participants described distressing and traumatic experiences associated with epilepsy symptoms. These included serious and at times life-threatening injuries during seizures; ' $I$ ended up with a cracked vertebra and a fractured skull.' (Christine). Following a seizure subsequent healthcare experiences, particularly hospitalisations were also distressing and appeared to contribute to a fear of subsequent epileptic attacks. Epilepsy had a powerful presence and participants often described themselves feeling vulnerable and helpless; ' $I$ was in a bed state. I asked someone to take me to the bathroom and of course they were too busy, so I finished up on all fours...' (Susan). The adverse consequences of seizures also included a strong concern around the impact on others, particularly loved ones, who seemed to share similar anxieties and fears during epileptic attacks; 'It's frightening for them, he (husband) hadn't experienced anything like this before so he was anxious' (Jennifer).

\subsection{2 'It's an ever-present thing, is the epilepsy'}

Almost every participant described epilepsy as chronic and incurable; 'I don't see that they're going to find any drugs or any level of drugs that are going to cure it completely.' (Carol). Some explained that being at a later stage of life made them less hopeful that a medical treatment would cure or manage their symptoms more successfully, 'I am 81, most likely won't live many more years and eh, I think is going to be there all the time now.' (Alfred). Epilepsy had a constant presence and an unpredictable and erratic nature. As a result, people often found themselves anticipating and worrying about the next attack; 'very often I'm looking in the corner and thinking, that feeling I've got is this the beginnings of, of an event?' (Alan). Even when seizures were well controlled by medication, epilepsy still had a presence 
lurking in the background; 'Yes, I haven't had any seizures since I took err these tablets that were recommended but it is there in the background' (Beth).

\section{2 'They say you can live a normal life but you can't...'}

This superordinate theme illustrated psychosocial difficulties, losses and restrictions encountered as a result of living with the condition and was supported by three subordinate themes.

\subsubsection{The impact of society's attitudes}

Epilepsy was described as a socially stigmatising condition. The stigma appeared to resonate in negative lay beliefs about epilepsy. Participants described how negative attitudes and societal ignorance made them feel ostracised and isolated; '...most people have their own ideas about it. They think you're (laughs), you know, crazy and eh, that's awful' (Alfred). There were various instances where participants felt discriminated and excluded from social forums because of their condition; 'Suddenly people in a Christian surrounding, didn't want to have anything to do with you' (Ian). Consequently, many kept their diagnosis concealed in an attempt to avoid shame and social exclusion; '...the other residents for example would cross the street to get away from me. (...) after that happened, I vowed I wouldn't tell anybody' (Susan). Even participants themselves appeared to have a fragmented understanding of their condition; 'I don't know I don't know how it all works' (Jennifer). The concealment itself appeared to feed the stigma and limited understanding both at an individual and a societal level; 'I don't speak to people about it so maybe that's the problem' (Alan)

\subsubsection{Loss of control}

Participants described a loss of control as epilepsy was taking over aspect of their lives. The most apparent instance where this occurred was during a seizure. Seizures were described as a disconnection from the environment and an absence from the world where participants lost control of their body; 'It was the oddest sensation because I was here while the incident was going on. But it wasn't me that was laid on the floor.' (Christine). Loss of control over mind and body was also evident in the descriptions of the aftermath of a seizure; 'the worst part of having a seizure is not the seizure itself, that's the bit that's alright, it's afterwards (...) everything is a bit like a balloon that's burst' (Ian). Antiepileptic medication also had a similar effect; 'I had all kinds of medicine, two or three, four times a day. It was a case of shake me, I rattle.' (Susan). Participants also made references to a loss of control due to the multiple restrictions they faced because of epilepsy such as not being able to go out alone or avoiding situations that could affect their symptoms; 'It restricts you (....) you've got to watch so many things that you know affect you for a start.' (Ella).

\subsubsection{Dependence}

Dependence was a subtheme that was characterised by reliance on other people and medication. Many participants described a loss of independence due to epilepsy across different areas of their lives. Participants often relied on partners to be able to engage in everyday activities 'I usually do most of my heavy baking with the oven on when (husband) is around.' (Ella). Driving was an important loss for many and depending on other people for transport was difficult both on a practical and emotional level; 'If we are going out with friends then they have to be the drivers and I have to be passenger.' (Carol). Finally, dependence on medication was prominent in the narratives. Participants described that without medication there was a lot of uncertainty and fear about recurrent seizures; 'If I forgot to take me tablets I don't know what would happen' (Alfred). 


\subsection{Attempts to adjust and cope}

This superordinate theme illustrated parallel coping strategies and processes aiming to facilitate acceptance of epilepsy and adjustment to its psychosocial impact. There were three subordinate themes reflecting these processes.

\subsubsection{Desire for acceptance and understanding}

In response to feelings of discrimination and exclusion participants described a desire for acceptance and understanding operating at two levels. The first level was the need for more acceptance of the condition in society through increased awareness and compassion from the general public and healthcare professionals; 'I think people should be there to help and err make sure you can live your life normal, or as normal as you can really'. (Jennifer). Some participants attempted to do this by actively reaching out for support and tried to escape the social isolation caused by epilepsy stigma; 'I go out of the house and I meet the rest of the world and that in fact is a therapy in itself' (Ian). The second level was an internal process where participants described attempts to make sense of and understand their condition. This process was characterised by seeking information from a variety of sources including healthcare professionals, medical investigations, research, social media, reflective thinking and sharing experiences with others; 'when you come to terms with epilepsy you come to understand it, investigation gives you better understanding of it, so I can say I could spend two hours on a computer screen reading up epilepsy' (Ian). Several participants emphasised the importance of a space to talk about their concerns related to epilepsy, particularly during appointments with the specialist epilepsy nurse, to help facilitate a process of adjustment; 'if I had some more (Epilepsy Nurse's name) pastoral care sort of err attention, I think things would have ended sooner and err and I could have been helped there because it would make me analyse what was going on rather than just cope.' (Beth).

\subsubsection{Taking back control}

In an attempt to battle the loss of control, participants were learning to live with the condition in the context of the restrictions and difficulties they faced. This theme was characterised by a wide range of helpful attitudes and coping strategies that allowed participants to regain a sense of control. Across all participants' accounts there was a clear sense of resilience and strength with many articulating a strong determination to manage the challenges of epilepsy, 'I believe in getting up and getting out and doing what you can.' (Susan). Some tracked this resilience in their life-story and legacy, 'It runs in the family and it runs in Yorkshire (...) It's not stopping me I am going out' (Christine). This strong determination encouraged several self-management and self-care strategies including diet, sleep, emotional expression and practical adaptations. For some these strategies were not only aiming to manage epilepsy but were also an integral part of maintaining well-being in later life, '...you've got to eat well, you've got to live a healthy lifestyle, you've also got to be able to get plenty of sleep (Ian). Participants described conflicting thoughts about their medication. At times, it was described as a way to control and manage epilepsy symptoms 'I think I have total control now I have this Tegrotol pills.' (Beth). However, it was also perceived as a cause of difficult side effects that caused them to feel out of control.

\subsubsection{We are in this together}

In contrast to 'dependence', this theme was characterised by supportive and collaborative relationships between participants' significant others and their epilepsy nurse. The experience of epilepsy was often shared with participants and their partners who united to manage the condition. Throughout the accounts participants often used 'we' instead of 'I' to describe 
their experiences. There was a close empowering bond between couples, for those who were in a relationship; 'But we've got each other and that helps.' (Beth). For some, the condition even strengthened these relationships 'I think I am closer with (husband) because of it than I would be probably in an ordinary marriage kind of thing, you know' (Ella). This strong connection was also evident during the interview process as many participants invited their partners to share their side of the story. At times, they would even finish each other's sentences; 'it is nice having (husband's name) to sort of, I feel it's nice you can correct me on things I have forgotten, or eh help to explain things more thoroughly.' (Carol). A close, supportive and collaborative relationship was also evident between participants and their specialist epilepsy nurse. This relationship was based on trust and mutual respect.

Participants felt valued as a person rather than just as a patient; 'Just having her in the background and on my side and really concerned about me getting better, not just controlling the epilepsy, you know' (Beth). The epilepsy nurse was viewed as a valuable ally who would provide collaborative and holistic care plans incorporating medical and psychosocial elements 'Yeah, you know I can talk to her like I can talk to you.' (Sarah)

\section{Discussion}

Older people living with epilepsy conceptualised their condition as powerful, persistent and unpredictable. The threatening nature of epilepsy was shaped by physically and emotionally traumatic experiences associated with seizures and their aftermath. The power of the condition was driving a struggle to balance negative psychosocial consequences with parallel coping and adjustment mechanisms. In the wider epilepsy population seizures pose serious and at times life-threatening physical injuries [27] whilst the frequency, severity and volatility of seizures is a significant predictor of depression and anxiety [28, 29]. Similarly, the unpredictability and constant presence of epilepsy symptoms appeared to fuel anxiety in relation to subsequent attacks for participants in this study.

These experiences are likely to contribute to a strong and negative illness identity. Studies on the CSMIR in epilepsy and other health conditions show that a negative illness identity is associated with 'unhelpful' coping such as avoidant strategies and poor psychological wellbeing [19, 20, 21]. Furthermore, older people's perceptions of epilepsy as incurable, chronic and difficult to control can also have a negative impact on coping, health and psychosocial outcomes [19]. Indeed, the perceived power of epilepsy appeared to be driving the negative consequences on quality of life and pose a barrier to helpful coping strategies and adjustment processes. However, the relationship between illness beliefs, coping, psychosocial and health outcomes is dynamic in nature [21]. Psychological interventions may help to create a positive shift in these associations, through processing distressing and traumatic experiences associated with health threats and diffuse negative illness representations [30]. Psychotherapeutic input in epilepsy patients can also facilitate adjustment, reinforce self-management, improve quality of life, and reduce depression, suicidal ideation and anxiety [31-35].

The impact of stigma was a critical concern for participants in this study. Stigma is common in younger and older adults living with epilepsy and is associated with higher levels of depression, anxiety and poorer quality of life [7, 12, 28, 36-38]. Older people with epilepsy may be more susceptible to stigmatising attitudes than their younger counterparts as their beliefs about epilepsy may be influenced by negative socio-cultural beliefs determined by their generational circumstances $[39,40]$. Furthermore, they may experience 'double stigma' from both epilepsy and negative societal attitudes towards ageing [41,42]. Concealment and 
nondisclosure of the diagnosis employed to avoid discrimination and stigma may limit opportunities to seek social support and contribute to isolation which in-turn can reinforce the cycle of stigma [12,37]. It is also likely to prevent individuals from obtaining information to inform a coherent understanding of their condition, which is an important aspect in forming helpful and accurate illness beliefs [16,17]. The process of concealment may account for participant's fragmented understanding and misconceptions about epilepsy and the desire for further knowledge about their condition, both of which have also been emphasised in previous work [37].

These findings highlight the need to target stigma and lack of awareness surrounding epilepsy. National and global health campaigns can target stigma at a societal level [38, 43]. At an individual level, healthcare provision needs to consider routine assessment and tailored interventions for misconceptions about epilepsy, stigma and its impact on older people. This could be achieved through staff training, support groups, and self-management and educational programmes. Such interventions have been associated with a reduction in perceived stigma, anxiety and visits to emergency departments, as well as improvement in psychological wellbeing, quality of life, self-efficacy and optimisation of coping strategies $[37,44-47]$.

Loss of control and independence were also prominent psychosocial challenges caused by seizures, antiepileptic medication side effects and restrictions on activities of daily living. These were met with parallel attempts to restore control. The process of losing and restoring perceived control has a critical role in adjustment for adults experiencing their first seizure [48]. In this study, participants had a diagnosis for more than two years. This suggests that adjustment trajectories may be a continuous process in the experience of epilepsy across the lifespan. The continued battle to restore control appeared to be driven by traumatic and pervasive experiences associated with seizures, a finding that is also supported by previous research [48]. A persistent loss of control is associated with fears about mortality and vulnerability [48]; issues that can be particularly relevant in later life. Older people living with epilepsy are faced with parallel restrictions and losses associated with ageing including changes in physical health, bereavements, loss of independence and loss of role [11, 39]. These parallels may intensify psychosocial challenges for people living with the condition in later life [11].

The internal strategies employed to restore control and independence included cognitive and emotional strategies like determination, positive attitudes and reflections on personal resilience. These mirror previous findings that older people derive hope and find positive ways of coping with other chronic neurological conditions such as dementia [49]. They also employed active behavioural coping strategies such as problem-solving, self-care and medication adherence; strategies that have been associated with lower anxiety and depression in the general epilepsy population [50]. Interventions encouraging self-management strategies [32] can maintain helpful coping and psychological well-being and may help to restore a perceived sense of control [48].

External coping strategies such as collaborative and supportive relationships with partners and the specialist epilepsy nurse had a vital role in coping and adjustment. Social and family support is recognised as integral in coping with epilepsy and other chronic health conditions $[51,52]$. Supportive marital relationships can minimise the psychological impact of stressors in later life [53]. It is therefore important to shape relationship-centred models of care in the assessments and healthcare provision for older people with epilepsy and encourage 
involvement with social supportive networks. The supportive and collaborative relationship with specialist epilepsy nurses was also very important in the management of epilepsy. Interactions with the specialist nurse were characterised by opportunities to process difficult experiences and get information on medical and psychosocial issues. Previous research has also shown that people with epilepsy highly value the support and interventions that specialist nurses provide, particularly in relation to psychosocial wellbeing, information provision and facilitation of self-management and medication adherence [44, 45, 54-57]. These findings provide strong support for the importance of specialist nursing in comprehensive healthcare provision in epilepsy for this age group.

\subsection{Limitations and future work}

There are methodological limitations that should be considered when interpreting the results of this study. The idiographic nature of the participant sample is compromised by differences in time of diagnosis between participants. Even though the impact of age at diagnosis is beyond the scope of this paper, there is evidence to suggest that older people diagnosed after the age of 65 have a better psychosocial functioning than people diagnosed at earlier stages in their life [6]. More work is required to understand what factors influence these differences and provide an insight into aspects influencing adjustment, coping and illness perceptions at different life stages and time trajectories. Furthermore, we have employed a culturally homogeneous sample of older people who were predominantly White British. IPA looks into the phenomenology and lived experience of different events and phenomena which are likely to be influenced by cultural factors. Further work should therefore investigate experiences of epilepsy in older people from other ethnic backgrounds. Participant recruitment was on a voluntary basis from patients who were actively engaged with the Neurology department. This suggests that respondents were willing to talk about their condition. The experiences of older people who may be more stigmatised and isolated may not be represented in this study. Our findings have also highlighted the importance of relationships in coping with epilepsy in later life. Further research can provide more insight into the shared experience of the condition within families and carers through interviewing dyads. It will also be useful to look further into the lived experience of epilepsy in older people who have limited social support to identify their needs and subsequently shape healthcare interventions.

\subsection{Conclusion}

Our findings have highlighted threatening cognitive representations held by older people living with epilepsy. These representations are shaped by the high prevalence of distressing experiences associated with the persistent and erratic nature of epilepsy symptoms. There are various psychosocial difficulties experienced in this age group including stigma, loss of control and loss of independence. The emotional sequel of these challenges may be amplified by parallel difficulties associated with ageing. Older people experience a constant struggle to balance the consequences of their condition with co-existing internal and external coping strategies. Healthcare provision needs to employ routine psychosocial assessments and intervention to address challenges, misconceptions and stigma and strengthen helpful coping. There is large scope for psychologically informed interventions for this patient group including psychotherapy, relationship-centred models of healthcare, self-management and educational programmes, and support groups. These can encourage helpful coping strategies, strengthen supportive networks, provide opportunities to process traumatic experiences, shape positive illness beliefs and address stigma at an individual level. Accessible national and global campaigns are also needed to target stigma at a societal level. Finally, the role of the epilepsy nurse is vital in providing holistic interventions to target the multitude of biopsychosocial issues experienced by older people with epilepsy. 


\section{Acknowledgments}

This study was supported by The University of Hull and Humber NHS Foundation Trust as part of an approved doctorate research dissertation. We would like to thank our participants and staff at the Neuroscience Department at a regional hospital who made this research possible.

\section{Conflict of Interest}

The authors confirm that there is no conflict of interest to declare.

\section{References}

[1] Kotsopoulos IAW, Van Merode TV, Kessels FGH, De Krom MCTFM, Knottnerus AJ. Systematic review and meta-analysis of incidence studies of epilepsy and unprovoked seizures. Epilepsia 2002;43:1402-9.

[2] Lhatoo SD, Johnson AL, Goodridge DH, MacDonald BK, Sander JW, Shorvon SD. Mortality in epilepsy in the first 11 to 14 years after diagnosis: multivariate analysis of a long-term, prospective, population-based cohort. Ann Neurol 2001;49:336-44.

[3] Brodie MJ, Elder AT, Kwan P. Epilepsy in later life. Lancet Neurol 2009;8:1019-30.

[4] Ramsay RE, Rowan AJ, Pryor FM. Special considerations in treating the elderly patient with epilepsy. Neurol 2004;62:24-9.

[5] Jacoby A, Baker GA. Quality-of-life trajectories in epilepsy: a review of the literature. Epilepsy Behav 2008;12:557-71.

[6] Baker GA, Jacoby A, Buck D, Brooks J, Potts P, Chadwick DW. The quality of life of older people with epilepsy: findings from a UK community study. Seizure 2001;10:92-9.

[7] McLaughlin DP, Pachana AN, Mcfarland K. Stigma, seizure frequency and quality of life: The impact of epilepsy in late adulthood. Seizure 2008;17:281-7.

[8] Laccheo I, Abla E, Heinrichs R, Sadler T, Baade L, Liow K. Assessment of quality of life among the elderly with epilepsy. Epilepsy Behav 2008;12:257-61.

[9] Haut SR, Katz M, Masur J, Lipton RB. Seizures in the elderly: Impact on mental status, mood and sleep. Epilepsy Behav 2009;14:540-4.

[10] Mclaughlin DP, Pachana NA, Mcfarland K. The impact of depression, seizure variables and locus of control on health related quality of life in a community dwelling sample of older adults. Seizure 2010;19:232-6.

[11]Baker GA Jacoby A. Quality of Life in Epilepsy: beyond seizure counts in assessment and treatment. Reading: Harwood Academic Press; 2000.

[12] Sleeth C, Drake K, Labiner DM, Chong J. Felt and enacted stigma in elderly persons with epilepsy: A qualitative approach. Epilepsy Behav 2016;55:108-12. 
[13]Pugh MV, Copeland LA, Zeber JE, et al. The Impact of Epilepsy on Health Status among Younger and Older Adults. Epilepsia 2005;46:1820-7.

[14] Martin R, Vogtle L, Gilliam F, Faught E. What are the concerns of older adults living with epilepsy? Epileps Behav 2005;7:297-300.

[15]Rapport F, Clement C, Doel MA, Hutchings HA. Qualitative research and its methods in epilepsy: Contributing to an understanding of patients' lived experiences of the disease. Epilepsy Behav 2015;45:94-100.

[16]Leventhal H, Nerenz DR, Steele DS. Illness representations and coping with health threats. In: Baum A, Taylor SE, Singer JE. Handbook of psychology and health. Hillsdale, NJ: Erlbaum; 1984.

[17]Leventhal H, Benyamini Y, Brownlee S, et al. Illness representations: Theoretical Foundations. In: Petrie, KJ, Weinman, J. Perceptions of health and illness: Current research and applications. Amsterdam: Harwood Academic Publishers; 1997.

[18] Moss-Morris R, Weinman J, Petrie KJ, Horne R, Cameron LD, Buick, D. The revised illness perception questionnaire (IPQ-R). Psychol Health 2002;17:1-16.

[19] Hagger M, Orbell S. A meta analytic review of the common-sense model of illness representations. Psychol Health 2003;18:141-84.

[20]Kemp S, Morley S, Anderson E. Coping with epilepsy: do illness representations play a role? Br J Clin Psychol 1999;38:43-58.

[21] Goldstein LH, Holland L, Soteriou H. Mellers JDC. Illness representations, coping styles and mood in adults with epilepsy. Epilepsy Res 2005;67:1-11.

[22] Smith JA, Flowers P, Larkin M. Interpretative phenomenological analysis: Theory, method and research. London: Sage; 2009.

[23] Brocki JM, Wearden AJ. A critical evaluation of the use of interpretative phenomenological analysis (IPA) in health psychology. Psychol Health 2006;21:87-108.

[24] Smith JA. Qualitative Psychology: A practical guide to methods. London: Sage Publications; 2003.

[25] Carradice A, Shankland MC, Beail N. A qualitative study of the theoretical models used by UK mental health nurses to guide their assessments with family caregivers of people with dementia. Int J Nurs Stud 2002;39:17-26.

[26] Harman G, Clare L. Illness representations and lived experience in early-stage dementia. Qual Health Res 2006;16:484-502.

[27]Nguyen R, Téllez-Zenteno, J. Injuries in epilepsy: a review of its prevalence, risk factors, type of injuries and prevention. Neurol Int 2009;1:e20. 
[28] Reisinger EL, Dilorio C. Individual, seizure-related, and psychosocial predictors of depressive symptoms among people with epilepsy over six months. Epilepsy Behav 2009;15:196-201.

[29] Vazquez B, Devinsky O. Epilepsy and anxiety. Epilepsy Behav 2003;4:20-25.

[30]Crossley ML. Narrative psychology, trauma and the study of self/identity. Theory Psychol 2000;10:527-46.

[31]Dewhurst E, Novakova B, Reuber M. A prospective service evaluation of acceptance and commitment therapy for patients with refractory epilepsy. Epilepsy Behav. 2015;46:23441.

[32] Edward K, Cook M, Giandinoto J. An integrative review of the benefits of selfmanagement interventions for adults with epilepsy. Epilepsy Behav 2015;45:195-204.

[33] Crail-Meléndez D, Herrera-Melo A, Martínez-Juárez IE, Ramírez-Bermúdez J. Cognitive-behavioral therapy for depression in patients with temporal lobe epilepsy: A pilot study. Epilepsy Behav 2012;23:52-6.

[34] Macrodimitris S, Wershler J, Hatfield M, et al. Group cognitive-behavioral therapy for patients with epilepsy and comorbid depression and anxiety. Epilepsy Behav 2011;20:83-8.

[35] Gandy M, Sharpe L, Perry KN. Cognitive behavior therapy for depression in people with epilepsy: A systematic review. Epilepsia 2013;54:1725-34.

[36] Scambler G, Hopkins A. Being epileptic: coming to terms with stigma. Sociol Health Illn 1986;8:26-43.

[37]Kilinc S, Campbell C. 'It shouldn't be something that's evil, it should be talked about': A phenomenological approach to epilepsy and stigma. Seizure 2009;18:665-71.

[38] Morrell MJ. Stigma and epilepsy. Epilepsy Behav 2002;3:21-5.

[39] Knight B. Psychotherapy with older adults. $2^{\text {nd }}$ ed. Thousand Oaks, CA: Sage Publications, Inc; 1996.

[40] de Boer H, Mula M, Sander JW. The global burden and stigma of epilepsy. Epilepsy Behav 2008;12:540-6.

[41] Stirling E. Valuing older people: Positive Psychological Practice. Chichester: WilleyBlackwell; 2010.

[42]Butler RN. Age-ism: Another form of bigotry. Gerontol 1969;9:243-6.

[43] Gutteling JM, Seydel ER, Wiegman O. Previous experiences with epilepsy and effectiveness of information to change public perception of epilepsy. Epilepsia 1986;27:739-45. 
[44] Noble AJ, Morgan M, Virdi C, Ridsdale L. A nurse-led self-management intervention for people who attend emergency departments with epilepsy: the patients' view. J Neurol 2013;260:1022-30.

[45] Helde G, Bovim G, Brathen G, Brodtkorb E. A structured, nurse-led intervention program improves quality of life in patients with epilepsy: a randomized, controlled trial. Epilepsy Behav 2005;7:451-7.

[46]Elafros MA, Mulenga J, Mbewe E, et al. Peer support groups as an intervention to decrease epilepsy-associated stigma. Epilepsy Behav 2013;27:188-92.

[47]Fernandes PT, Snape DA, Beran RG, Jacoby A. Epilepsy stigma: what do we know and where next? Epilepsy Behav 2011;22:55-62.

[48] Velissaris SL, Wilson SJ, Saling MM, Newton MR, Berkovic SF. The psychological impact of a newly diagnosed seizure: Losing and restoring perceived control. Epilepsy Behav 2007;10:223-33.

[49] Wolverson Radbourne E, Clarke C, Moniz-Cook E. Remaining hopeful in early-stage dementia: a qualitative study. Aging Ment Health 2010;14:450-60.

[50]Livneh H, Wilson, LM, Duchesneau A, Antonak RF, 2001. Psychosocial adaptation to epilepsy: the role of coping strategies. Epilepsy Behav 2001;2:533-44.

[51]Elliott JO, Charyton C, Sprangers P, Lu B, Moore JL. The impact of marriage and social support on persons with active epilepsy. Epilepsy Behav 2011;20:533-8.

[52] Martire LM, Lustig AP, Schulz R, Miller GE, Helgeson VS. Is it beneficial to involve a family member? A meta-analysis of psychosocial interventions for chronic illness.

Health Psychol 2004;23:599-611.

[53]Bookwala J, College L. Marital quality as a moderator of the effects of poor vision on quality of life among older adults. J Gerontol Ser B: Psychol Sci Soc Sci 2011;66:60516.

[54] Mills N, Bachmann MO, Harvey I, Hine I, McGowan M. Effect of a primary-care-based epilepsy specialist nurse service on quality of care from the patients' perspective: quasiexperimental evaluation. Seizure 1999;8:1-7.

[55]Betts T, Greenhill L. The cost of everything and the value of nothing. Seizure 2001;10:628-32.

[56]Ridsdale L, Kwan I, Morgan M. How can a nurse intervention help people with newly diagnosed epilepsy? A qualitative study of patients' views. Seizure 2003;12:69-73.

[57]Hosking PG. The specialist nurse role in the treatment of refractory epilepsy. Seizure 2004;13:303-7. 


\section{Appendices}

\section{Appendix A - Semi-structured Interview Schedule ${ }^{2}$}

I would like to ask you some questions about your epilepsy. A lot of people with this condition have different opinions and experiences. I am interested to know about your experience and beliefs about your epilepsy.

\section{Can you tell me your story and how you started attending this clinic?}

- What are the reasons that you visit this clinic?

- What made you realise that something was wrong? How/ when did you realise that something was wrong?

- How would you describe epilepsy to other people?

\section{How long do you think the epilepsy will last?}

- How do you think your epilepsy will be like in one year's time? What changes do you anticipate?

\section{How does epilepsy affect your life?}

- What is the impact of epilepsy in your life?

- How did epilepsy change your life?

- What are the most difficult effects/ consequences of your epilepsy?

- Can you tell me about any good effects/ consequences of your epilepsy?

4. How much control do you feel that you have on the epilepsy?

- How do you think the epilepsy can be treated?

- What/ who can help you manage/control the epilepsy?

5. What do you think is the cause of the epilepsy?

- How do you think the epilepsy started?

6. Is there anything else that you would like to say?

- Is there anything else that would be helpful for me to know?

- How did you find this interview?

\section{Examples of general prompts:}

- Can you tell me a bit more about that?

- Earlier you mentioned (....) what was that like for you?

\footnotetext{
2 The Interview Schedule was adapted from:

Green A, Payne S, Barnitt R. Illness representations among people with nonepileptic seizures attending a neuropsychiatry clinic: a qualitative study based on the self-regulation model. Seizure 2004;13:331-9. 